1 H. MAecker, Z. Naturforsch. 11 a, 457 [1956].

2 H. Maecker u. S. Steinberger, Z. Angew. Phys. 23, 456 [1967].

3 V. R. Watson u. E. B. Pegot, NASA TN D-4042 [1967].

4 P. S. Masser, Arc jet design, ARS Paper 2352-62 [1962].

5 K. Oswatitsch, Gasdynamik II. 8. Springer-Verlag, Wien 1952.

6 L. Niemeyer u. K. Ragaller, AIAA Paper No 72/415.

7 H. Schardin, Ergebn. Exakt. Naturwiss. 20, 303 [1942].

8 M. Born u. E.Wolf, Principles of Optics, Pergamon Press, 3rd edition 1965, $121 \mathrm{ff}$.

9 U. Kogelschatz u. W. R. Schneider, Z. Angew. Math. Phys. 21, 666 [1970].

10 H. Magdeburg u. M. Schley, Z. Angew. Phys. 20, 465 [1966].

11 W. FrIE, Ann. Phys. 10, 332 [1962].
12 W. FrIE, private Mitteilung.

$13 \mathrm{H}$. W. Drawin u. P. Felenbok, Data for Plasma in Local Thermodynamic Equilibrium, Paris, Gauthier-Villars 1965.

14 W. L. Wiese, M. W. Smith u. B. M. Glenon, Atomic Transition Probabilities, Vol. I, NSRDS-NBS 4 [1966].

15 C.E. Moore, Atomic Energy Levels, Vol. I, NBS 467 [1949].

16 H. R. Griem, Plasma Spectroscopy, Mc-Graw-Hill Book Co. 1964.

17 K. H. Wilson u. W. E. Nicolet, J. Quant. Spectr. Radiative Transfer 7, 891 [1967].

18 G. Boldt, Z. Phys. 154, 330 [1959].

19 J. C. Morris, R. U. Krey u. R. L. Garrison, Phys. Rev. 180, 167 [1996].

20 W. Hermann u. E. Schade, J. Quant. Spectr. Radiative Transfer 12, 1257 [1972].

\title{
Determination of Plasma Electron Temperature from the Reversal of Radial Ambipolar Electric Field in a Longitudinal Magnetic Field
} GÜNTER FRANCK

\author{
OSRAM-Studiengesellschaft München \\ R. HELD and H.-D. PFEIL \\ Sektion Physik der Universität München \\ (Z. Naturforsch. 27 a, 1439-1443 [1972] ; received 8 May 1972)
}

\begin{abstract}
When a longitudinal magnetic field is superimposed to a low pressure plasma, reversal of the radial ambipolar electric field takes place at a definite magnetic field $B_{\mathrm{r}}$. From the $B_{\mathrm{r}}$-values electron temperatures $T_{\mathrm{e}}$ in the plasma can be found, when classical magnetic field diffusion exists.

In the negative $\mathrm{H}_{2}$ - and He-glow plasmas with superimposed longitudinal magnetic field, electron temperatures have been measured on the one hand by the method of electric field reversal and on the other hand with Langmuir probes. There is a fairly good agreement between the $T_{\mathrm{e}}$-values obtained by both methods.
\end{abstract}

\section{Method}

If in a cylindrical plasma column with superimposed longitudinal magnetic field ambipolar radial plasma currents to the wall and classical electron-neutral and ion-neutral diffusion can be assumed, the radial electric field $E_{\perp}$ in the column is given by (see e. g. ${ }^{1}$ )

$$
E_{\perp}=-\frac{D_{\mathrm{e} \perp}-D_{+\perp}}{b_{\mathrm{e} \perp}+b_{+\perp}} \frac{1}{n_{\mathrm{e}}} \frac{\mathrm{d} n_{\mathrm{e}}}{\mathrm{d} r}
$$

with

$\begin{aligned} D_{\mathrm{e} \perp}=\frac{D_{\mathrm{e}}}{1+b_{\mathrm{e}}^{2} B^{2}}, & D_{+\perp}=\frac{D_{+}}{1+b_{+}{ }^{2} B^{2}}, \\ b_{\mathrm{e} \perp}=\frac{b_{\mathrm{e}}}{1+b_{\mathrm{e}}^{2} B^{2}}, & b_{+\perp}=\frac{b_{+}}{1+b_{+}{ }^{2} B^{2}} .\end{aligned}$

Reprint requests to Dr. G. FRANCK, OSRAM-Studiengesellschaft, $D-8000$ München 90, Hellabrunner-Str. 1.
$\left(D_{\mathrm{e} \perp}, D_{+\perp}\right.$ electron- and positive ion-diffusion coefficient across the magnetic field; $D_{\mathrm{e}}, D_{+}$electron- and ion-diffusion coefficient in the absence of a magnetic field; $n_{\mathrm{e}}$ plasma electron density; $b_{\mathrm{e} \perp}, b_{+} \perp$ electronand ion mobilities across the longitudinal field; $b_{\mathrm{e}}, b_{+}$ electron and ion mobility in the absence of a magnetic field; $B$ longitudinal magneitic field.)

In the absence of a magnetic field, the radial ambipolar field is positive, retarding plasma electrons and accelerating plasma ions, since $D_{\mathrm{e}} \gg D_{+}$.

Increase of the magnetic field from zero to higher values causes a decrease of $D_{\mathrm{e} \perp}$ and $D_{+}$in (1). Since at a given pressure the electron mobility $b_{\mathrm{e}}$ is larger than the ion mobility $b_{+}$by a factor of about $10^{2}$ to $10^{3}$, the electron diffusion coefficient $D_{\mathrm{e} \perp}$ is diminished much stronger than $D_{+} \perp$ with increasing magnetic field. This causes that with growing magnetic field $B$ the radial ambipolar electric field (1) vanishes at a special magnetic field $B_{\mathrm{r}}$. When $\quad D_{\mathrm{e} \perp}\left(B_{\mathrm{r}}\right)=D_{+\perp}\left(B_{\mathrm{r}}\right), \quad E_{\perp}=0$. 
For magnetic fields higher than $B_{r}$, the ambipolar electric field will become negative, accelerating the plasma electrons to the wall and retarding the plasma ions.

Using the Einstein relation $D_{\mathrm{e}}=b_{\mathrm{e}} U_{\mathrm{e}}, D_{+}=$ $b_{+} U_{+}$, and the condition $b_{+} U_{+} \ll b_{\mathrm{e}} U_{\mathrm{e}}$, from Eq. (3) we get a simple relation for the magnetic field, at which ambipolar electric field reversal takes place:

$$
B_{\mathrm{r}}=\frac{p}{\sqrt{\left(b_{+} p\right)\left(b_{\mathrm{e}} p\right)\left(U_{+} / U_{\mathrm{e}}\right)-\left(b_{+} p\right)^{2}}}
$$

( $p$ neutral gas pressure, $b_{\mathrm{e}} p, b_{+} p$ reduced electronand ion mobility).

From relation (4) the electron temperature of plasma electrons can be calculated easily provided that the dependence $b_{\mathrm{e}}\left(U_{\mathrm{e}}\right)$ is well known from other experiments. Then from (4) we find an implicit equation for $U_{\mathrm{e}}$ :

$$
U_{\mathrm{e}}=U_{+} \cdot b_{\mathrm{e}}\left(U_{\mathrm{e}}\right) p \cdot b_{+} p /\left[\left(p / B_{\mathrm{r}}\right)^{2}+\left(b_{+} p\right)^{2}\right] .
$$

This formula is only valid for classical ambipolar diffusion across the longitudinal magnetic field.

As an example we calculate $B_{\mathrm{r}}$ in the positive column in He. Assuming the parameters $p=0.5$ Torr, $U_{\mathrm{e}}=1 \mathrm{~V}, T_{+}=300 \mathrm{~K}, b_{+} p=8.1 \cdot 10^{3}$ Torr $\mathrm{cm}^{2} \mathrm{~V}^{-1}$ $\mathrm{s}^{-1}, b_{\mathrm{e}} p=0.8 \cdot 10^{6}$ Torr $\mathrm{cm}^{2} \mathrm{~V}^{-1} \mathrm{~s}^{-1}$ (these numerical values for reduced mobilities will be explained later), we get $B_{\mathrm{r}} \approx 3500$ Gauss. This magnetic field is much higher than the critical magnetic field, above which helical instabilities set in. In experiments ${ }^{2}$ the critical magnetic field in the positive $\mathrm{He}$ column for parameters similar to our example has been found to about 1500 Gauss. Recent measurements ${ }^{3}$ of the critical magnetic field in the rare gases $\mathrm{Ne}$, $\mathrm{Ar}, \mathrm{Kr}, \mathrm{Xe}$ also show that in most cases the magnetic field for ambipolar field reversal is much higher than the critical one. - Only for $0<B<B_{\mathrm{c}}$ classical ambipolar diffusion takes place, whereas at higher values $B>B_{\mathrm{c}}$ Kadomtsev-instabilities ${ }^{4}$ set in, enlarging the effective ambipolar diffusion with increasing magnetic field by $E \times B$ drift, which tends to drive the plasma electrons radially outwards and to amplify the diffusion.

Thus the method of ambipolar field reversal for determining $T_{\mathrm{e}}$ from (5) in the most cases is not available to the positive column, since the magnetic field which has to be applied for the field reversal is too high and lies above the critical magnetic field $B_{\mathrm{c}}$, where no classical diffusion exists.
In contrast to the positive column, the negative glow is a plasma with very low electron temperatures $\left(U_{\mathrm{e}}<0.1 \mathrm{~V}\right)$, so that the characteristic magnetic field $B_{\mathrm{r}}$, where ambipolar field reversal sets in, is much lower than in the positive column. In the negative glow at low pressures $p<0.5$ Torr the condition

$$
B_{\mathrm{r}}<B_{\mathrm{c}}
$$

is fulfilled, so that electron temperatures can be evaluated from (5) and compared with probe measurements.

\section{Experimental}

Ambipolar field and electron temperature measurements were carried out in the negative glow of anomalous glow discharges in $\mathrm{H}_{2}$ and $\mathrm{He}$. The distance between the water cooled Al-cathode and -anode is $30 \mathrm{~cm}$. There is no Faraday dark space and no positive column existent. Between the two water cooled coils for magnetic field generation up to 600 Gauss there is a slit of $1 \mathrm{~cm}$ for leading through two radially movable probes.

For electron temperature measurements four different radii of the discharge tube have been used: $R=0.94$, $1.2,1.8$ and $3 \mathrm{~cm}$. The electron temperature measurements have been carried out in the following manner. A platinum probe, $0.05 \mathrm{~mm}$ in diameter, was always held incandescent by electron impact. Measurement of $T_{\text {e }}$ takes place $30 \mathrm{sec}$ after turning off the high positive probe voltage in a time interval of about 5 sec. The probe voltage is measured versus a second directly heated floating probe. - The radial ambipolar electric field is obtained from radial floating potential measurements. The inversion point is found in the most sensitive way at a distance of about $6 \mathrm{~mm}$ to the wall. Here the magnetic field $B_{\mathrm{r}}$ is found with an accuracy of about 1 Gauss.

Up to magnetic fields of 600 Gauss no oscillations in the negative glow have been found from probe measurements.

\section{Results}

In Fig. $1 \mathrm{a}, \mathrm{b}$ the magnetic fields $B_{\mathrm{r}}$, at which reversal of the radial ambipolar electric field from positive to negative values takes place, and the derived electron temperatures are shown. The $T_{\mathrm{e}}(p)$ values are calculated from Eq. (5) with $U_{+}=U_{\text {gas }}$. The $U_{\mathrm{e}}$-dependent electron mobilities $b_{\mathrm{e}}\left(U_{\mathrm{e}}\right)$ in $\mathrm{H}_{2}$ and $\mathrm{He}$ are obtained from measurements of electron drift velocities as a function of the reduced electric field $E / p\left(\mathrm{H}_{2}:{ }^{5-9}, \mathrm{He}:{ }^{5,6}\right)$ and from measurements of the characteristic energy $D_{\mathrm{e}} / b_{\mathrm{e}}$ as a function of $E / p$ $\left(\mathrm{H}_{2}:{ }^{10,11}, \mathrm{He}:{ }^{12,13}\right)$. Thus the $U_{\mathrm{e}}$-dependent $b_{\mathrm{e}}\left(U_{\mathrm{e}}\right)$ 
can be represented in the energy range $0.01 \leqq U_{\mathrm{e}}$ $\leqq 1 \mathrm{~V}$ by the following expressions:

$\mathrm{H}_{2}: b_{\mathrm{e}} p=3.8 \cdot 10^{5} \cdot U_{\mathrm{e}}^{-0,66}$ Torr $\mathrm{cm}^{2} \mathrm{~V}^{-1} \mathrm{~s}^{-1}$,

He: $b_{\mathrm{e}} p=7.8 \cdot 10^{5} \cdot U_{\mathrm{e}}^{-0,64}$ Torr $\mathrm{cm}^{2} \mathrm{~V}^{-1} \mathrm{~s}^{-1}$.

These electron mobilities agree fairly well with the $b_{\text {e }} p$-values calculated from

$$
b_{\mathrm{e}} p=-\frac{e}{m} \frac{1}{N_{1}} \int_{0}^{\infty} \frac{1}{v \sigma_{\mathrm{m}}(v)} \frac{4 \pi v^{3}}{3} \frac{\partial F}{\partial v} \mathrm{~d} v
$$

$\left[\sigma_{\mathrm{m}}(v)\right.$ momentum transfer cross section, $N_{1}=3.22 \cdot 10^{16}$ $\mathrm{cm}^{-3}$ Torr $\left.^{-1}\right]$,

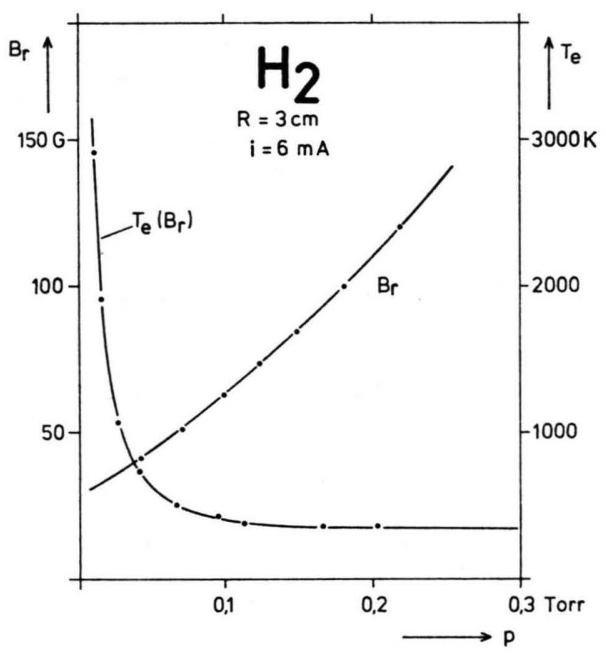

a

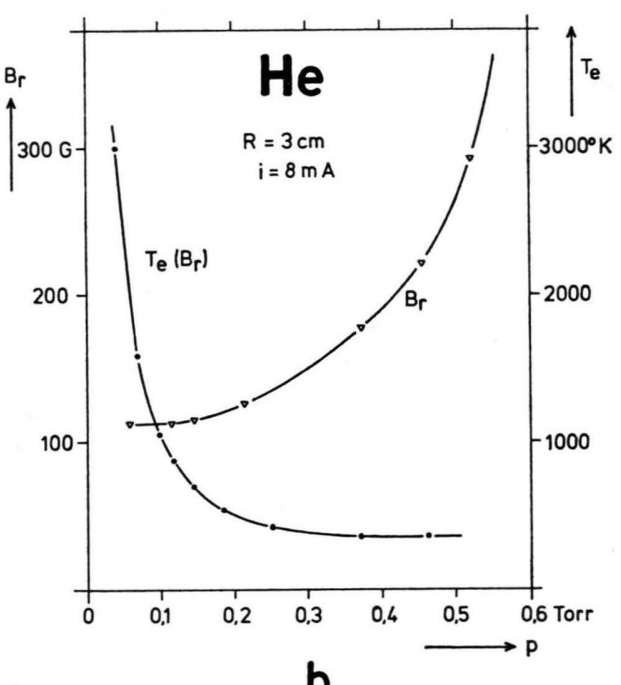

Fig. 1 a, b. Magnetic fields, at which reversal of the radial ambipolar electric field takes place and derived electron temperatures in the $\mathrm{H}_{2}$ - and He-negative glow. if for $F$ a Maxwellian velocity distribution is inserted and the momentum transfer cross sections in ${ }^{28}$ are used.

The ion mobilities are: $b_{+} p=9.3 \cdot 10^{3}$ Torr $\mathrm{cm}^{2} \mathrm{~V}^{-1}$

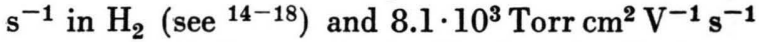
in $\mathrm{He}\left(\mathrm{see}^{19,20}\right)$. - In the He-glow the $\mathrm{He}_{2}{ }^{+}$-concentration can be neglected compared with the $\mathrm{He}^{+}$ions ${ }^{21}$. In the $\mathrm{H}_{2}$-glow the ion mobility probably corresponds to the predominating $\mathrm{H}_{3}{ }^{+}$-ion ${ }^{22,}{ }^{23}$.

\section{Comparison with Langmuir Probe Measurements}

The electron temperatures in Fig. 1 a, b obtained from these electron- and ion mobilities have to be compared with Langmuir probe measurements. The probe measurements have been carried out at different magnetic fields and discharge tube diameters. In Fig. 2 and Fig. 3 the electron temperatures obtained from probe measurements and from field reversal are plotted separately versus the reduced quantity $R p \cdot \sqrt{D_{\text {all }} / D_{\mathrm{a} \perp}}$ in $\mathrm{H}_{2}$ and He. $R$ is the tube radius and

$$
D_{\mathrm{a} \|} / D_{\mathrm{a} \perp}=1+b_{\mathrm{e}} b_{+} B^{2}
$$

$\left(D_{\mathrm{a} \perp}, D_{\mathrm{a} \|}\right.$ ambipolar diffusion coefficient perpendicular and parallel to the magnetic field).

The reduced quantity $R p \sqrt{D_{\mathrm{a}} / \mathrm{D} / D_{\mathrm{a}} \perp}$ generally is determining the electron temperature in a cylindrical low pressure plasma. This can be shown in the following simple manner: The important quantity for $T_{\mathrm{e}}$ is the ratio of mean diffusion losses $\bar{\nu}_{\text {loss }}$ to the elastic or inelastic collision losses $\bar{\nu}_{\mathrm{m}}$ of the plasma electrons. This ratio can be written in the following reduced form, supposing a zero order Bessel function for the radial plasma density distribution:

$$
\begin{aligned}
& \frac{\bar{\nu}_{\text {loss }}}{\bar{\nu}_{\mathrm{m}}}=\frac{1}{\bar{\tau}_{\text {diff }}} \frac{1}{\bar{\nu}_{\mathrm{m}}}=\left(\frac{R}{2,40}\right)^{2} \frac{1}{D_{\mathrm{a} \perp}} \frac{1}{\bar{\nu}_{\mathrm{m}}} \\
&=\left(\frac{R p}{2,40}\right)^{2} \frac{D_{\mathrm{a} \|}}{D_{\mathrm{a} \perp}} \frac{1}{\left(\bar{\nu}_{\mathrm{m}} / p\right)} \frac{1}{\left(D_{\mathrm{a} \|} \cdot p\right)} .
\end{aligned}
$$

For a given gas this ratio is a function of $R p \sqrt{D_{\mathrm{all}} / D_{\mathrm{a} \perp}}$, which therefore is the only relevant parameter of $T_{\mathrm{e}}$.

Figure 2 and 3 show that all electron temperatures obtained at different magnetic fields and tube diameters are reducible with reference to $R p \sqrt{D_{\mathrm{all}} / D_{\mathrm{a} \perp}}$. - The electron temperatures have been found independent on the discharge current.

Only at low electron temperatures there is discrepancy between probe measurements and the results 


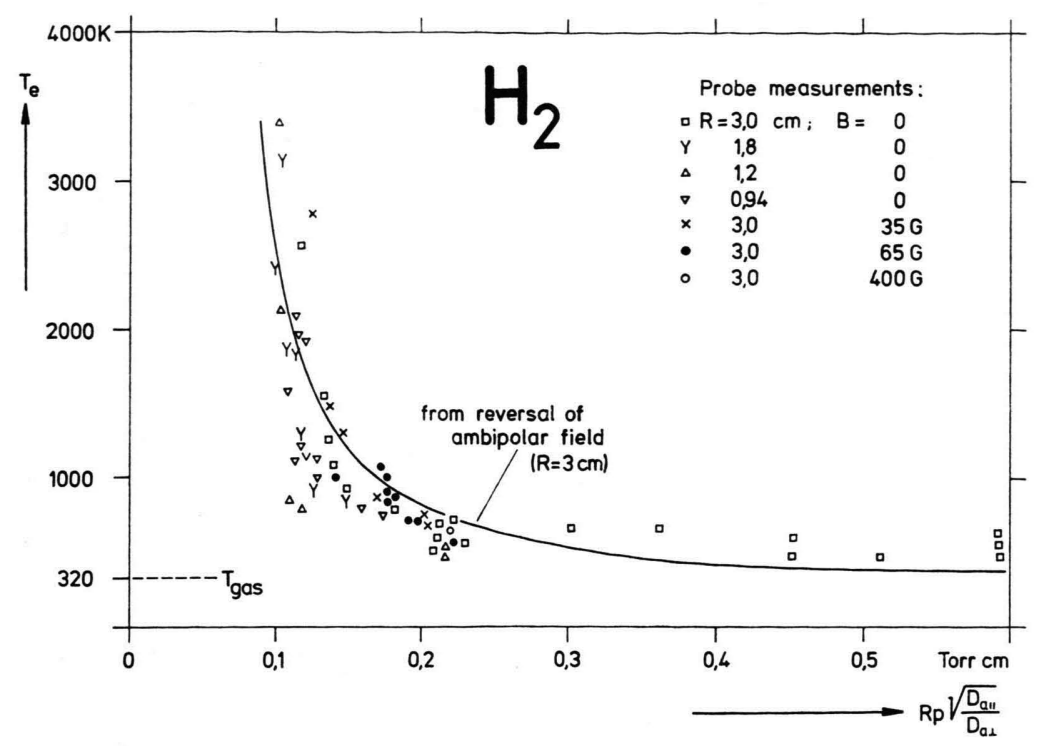

Fig. 2. Comparison of electron temperatures in the negative $\mathrm{H}_{2}$-glow from probe measurements and from the reversal of radial ambipolar electric field.

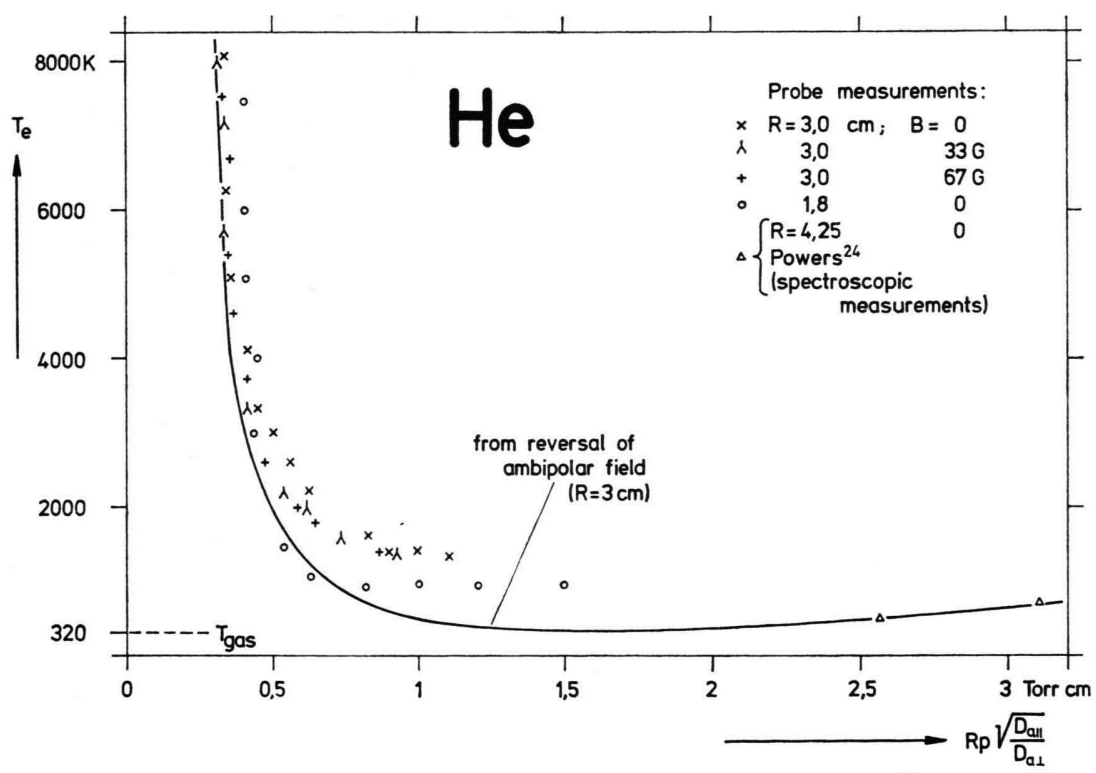

Fig. 3. Comparison of electron temperatures in the negative He-glow from probe measurements and from the reversal of radial ambipolar electric field.

of field reversal. Latter results we suppose to give the more correct values, since smallest impurities of the probe surface will cause higher apparent $T_{\mathrm{e}}$ values at these extremely low electron temperatures (work function, resistivity of surface layers), when the normal Langmuir probe method is used. At the field reversal method all these disturbing effects do not occur.
Probe measurements of other authors ${ }^{24-27}$ in the negative glow show similar low electron temperatures of about $500-600 \mathrm{~K}$ at higher pressures.

From radial probe measurements carried out at the magnetic field $B_{\mathrm{r}}$, we have found the $T_{\mathrm{e}}$-values independent on r, so that the comparision (Fig. 2,3) of probe measurements in the axis and field reversal measurements near the wall is correct. 
Our probe measurements of the axial electric field strenght show that there is a retarding field for electrons in direction of the anode $\left(E_{z} \approx 20 \mathrm{mV} / \mathrm{cm}\right)$. That means that the plasma electrons do not get energy from the field and can thermalize down to the temperature of the neutral gas $(\approx 320 \mathrm{~K})$.

Determination of electron temperature from electric field reversal is a very sensitive method in plasmas with low electron temperatures like the negative glow. Since in (5) the inequality $b_{+} p \ll p / B_{\mathrm{r}}$ is fulfilled in all cases, the degree of accuracy for $T_{\mathrm{e}^{-}}$

1 F. C. HoH, Rev. Mod. Phys. 34, 267 [1962].

2 F. C. HoH and B. LehNert, Phys. Fluids 3, 600 [1960].

3 G. JANZEN, F. Moser, and E. RÄUChLE, Z. Naturforsch. 25 a, 992 [1970].

4 B. B. Kadomtsev and A. V. Nedospasov, J. Nucl. Energy 1, 230 [1960].

5 J. L. PACK and A. V. Phelps, Phys. Rev. 121, 798 [1961].

6 J. L. Pack, R. E. Voshall, and A. V. Phelps, Phys. Rev. 127, 2084 [1962].

7 L. S. Frost and A.V. Phelps, Phys. Rev. 127, 1621 [1962].

8 A. G. Engelhardt and A.V. Phelps, Phys. Rev. 131, 2115 [1963] ; 133, 375 [1964].

9 J. J. Lowke, Austr. J. Phys. 16, 115 [1963].

10 R. W. Crompton and D. J. Sutton, Proc. Roy. Soc. London A 215, 467 [1952].

11 R. W. Crompton and M. T. Elford, VI. Int. Conf. Ioniz. Phen. in Gases, Paris 1963.

12 R. W. Crompton, M. T. Elford, and R. L. Jory, Austr. J. Phys. 20, 369 [1967].

13 L. M. Chanin, A.V. Phelps, and M. A. Biondi, Phys. Rev. 128, 219 [1962]. measurement from field reversal is given by the error of $B_{\mathrm{r}}$, which in our measurements was lower than 3 percent.

The method of field reversal is only available, if

1. plasma density and radial electric field in the plasma column can be described by the classical ambipolar diffusion model,

2. electron velocity distribution is nearly Maxwellian, so that transport coefficients $D_{\mathrm{e}}$ and $b_{\mathrm{e}}$ can be expressed as a function of $U_{\mathrm{e}}$.

14 L. M. Chanin, Phys. Rev. 123, 526 [1961]

15 G. JÄGER and W. OTto, Z. Physik 169, 517 [1962].

16 H. J. Osкam and V. R. Mittelstadt, Physica 30, 2021 [1964].

17 M. Saporoschenko, Phys. Rev. 139, 349 [1965].

18 G. Waidmann, Z. Physik 198, 548 [1967].

19 H. J. OsKam and V. R. Mittelstadt, Phys. Rev. 132, 1435 [1963].

20 J. A. HorNBECK, Phys. Rev. 84, 615 [1951].

21 D. Morris, Proc. Phys. Soc. London 68,11 [1955].

22 M. Schmidt, Beitr. Plasmaphysik 9, 227 [1969].

23 M. Novák and G. Waidmann, Z. Angew. Phys. 31, 300 [1971].

24 R. S. Powers, J. Appl. Phys. 37, 3821 [1966].

25 R. Mosburg, Jr., Phys. Rev. 152, 166 [1966].

26 J. M. Anderson, J. Appl. Phys. 31, 511 [1960].

27 W. STERN, Int. Conf. Phen. Ioniz. Gases, Vienna 1967, p. 87.

28 P. LABorie, J.-M. Rocard, and J. A. ReEs, Electronic Cross-Section and Macroscopic Coefficients, 1. Hydrogen and Rare Gases, Dunod, Paris 1968. 